\title{
High-Grade Pleomorphic Sarcoma of the Left Atrium after Incomplete Resection and Adjuvant Chemotherapy
}

\author{
Ricardo J. Razera, ${ }^{\circledR}$ Amanda Mendonça de Araújo, ${ }^{\circledR}$ Viviane Pereira Bernardes, ${ }^{\circledR}$ Fernanda Miranda Moisés, ${ }^{(0)}$ \\ Leonardo Santos Mundim, ${ }^{\circledR}$ Rogério A. Araújo ${ }^{\circledR}$ \\ Universidade Federal de Uberlândia - Faculdade de Medicina, Uberlândia, MG - Brazil.
}

\section{Introduction}

Primary cardiac tumors are extremely rare, found in only $0.02 \%$ of all autopsies. ${ }^{1}$ These tumors are malignant in only $25 \%$ of the cases, $75 \%$ of which are sarcomas, frequently affecting young people with no risk factors. ${ }^{2}$ The median overall survival is less than 1 year, ${ }^{3}$ ranging from 9 to 16 months, ${ }^{4,5}$ with rapid proliferation and deaths caused by arrhythmias, thrombosis, pulmonary embolism, ${ }^{6}$ and, most importantly, heart failure. ${ }^{7}$ Due to its rarity, reports in the literature about the best treatment are scarce, especially in malignant tumors. ${ }^{1}$ The aim of the present work is to report a case of an undifferentiated pleomorphic sarcoma (UPS) in the left atrium in which surgical treatment was associated with chemotherapy, with an overall and progression-free survival of neoplastic disease higher than the median described in previous manuscripts.

\section{Case Report}

A 32-year-old woman was admitted to the institution in May 2019, complaining of dyspnea upon exertion, which had lasted for 6 months and had progressively worsened, associated with sinus tachycardia, coughing, and vomiting. The patient underwent a Doppler echocardiogram, which showed a mass in the left atrium, with irregular contours, attached to the interatrial septum and involvement of the mitral valve, with an ejection fraction (Simpson) of 62\%; a double mitral lesion, with

\section{Keywords}

Cardiovascular Diseases; Neoplasms; Sarcoma/ complications; Sarcoma/surgery; Heart Atria/surgery; Atrial Function; Drug Therapy significant stenosis and moderate insufficiency; as well as tricuspid valve insufficiency and moderate pulmonary hypertension. It is important to note that the mass was not an artifact, since it was viewed in more than one slice.

Symptoms were attributed mainly to pulmonary congestion, caused by the predominance of stenosis, as compared to mitral regurgitation. Right (RA) and left (LA) atria presented a slight volumetric increase, the right ventricle $(\mathrm{RV})$ with a moderate increase in diameter and the left ventricle (LV) with a normal diameter. Slightly impaired biventricular function decreased to a slight degree only in the RV. The mitral valve apparatus contained a thickened anterior leaflet with reduced mobility and cupola due to the presence of tumor mass infiltration in the leaflet body, measuring approximately $18 \times 21 \mathrm{~mm}$ in diameter, coupled with a reduced valve opening, once again justifying the diagnosis of stenosis. The Doppler images showed a moderate, eccentric reflux to the LA lateral wall and a valve area of $0.7 \mathrm{~m}^{2}$. Tricuspid valve apparatus with slightly thickened leaflets was also observed, pulled by ventricular remodeling and presenting reduced mobility. Aortic and pulmonary valves showed no major changes, and the presence of diffuse pericardial effusion showed no major hemodynamic repercussions. The patient did not undergo a transesophageal echocardiogram, nor was the pericardial fluid evaluated.

The patient underwent surgery, conducting an incomplete resection of the mass in the interatrial septum and reconstruction with bovine pericardium, leaving a residual infiltrative lesion in the mitral valve, which was considered inoperable. Thus, this surgery was considered to be palliative to relieve the symptoms and to aid in the final diagnosis. Upon macroscopy, the surgical specimen revealed fragments of solid, infiltrative, 
yellowish-brown, and elastic tissue neoformation, with areas of non-encapsulated hemorrhaging. Histological sections showed fragments of poorly differentiated mesenchymal tissue neoformation, with high cellularity, comprised of spindle and epithelioid cells, with nuclear pleomorphism and moderate anaplasia, dispersed in a fibrillar or myxoid stroma, with up to 12 mitoses / 10 high-magnification fields (occasional atypical mitoses) and a tumor necrosis area (less than $50 \%$ of the neoplasia volume). Upon immunohistochemistry, histopathological analysis allowed us to conclude that it was a high-grade undifferentiated pleomorphic sarcoma. Staging exams showed disease restricted to the heart, and chest computed tomography (CT) showed a mass with soft tissue density and a lobulated contour located in the left atrium, measuring $6.0 \times 5.3 \mathrm{~cm}$ (Figure 1).

After incomplete tumor resection ( $\mathrm{R} 2$ surgery), the multidisciplinary team decided to start cytoreductive chemotherapy in an attempt to target the maximum response rate with subsequent reassessment for the second surgical procedure, if feasible, regarding the residual infiltrative lesions in pulmonary vessels. In this sense, the patient received three cycles of chemotherapy with ifosfamide and doxorubicin, followed by three cycles of ifosfamide alone. Doxorubicin was suspended at the end of the third cycle due to a drop in the left ventricular ejection fraction (LVEF) to $48 \%$. The patient is currently asymptomatic, using Carvedilol, Furosemide, and Enalapril, having been prophylactically anticoagulated only during the immediate postoperative period, as advised by the assistant cardiology team. She has a regular heartbeat with two sounds and a systolic murmur in the mitral focus $3+/ 6+$. Moreover, she is normotensive (blood pressure: $110 / 70 \mathrm{mmHg}$ ), eupneic (respiratory rate: 18 breaths per minute), with a heart rate of 62 beats per minute.

The revaluation tomography (Figure 2) showed a partial reduction in mass, but the echocardiogram quantified the lesions as stable after six cycles of chemotherapy, configuring a stable cancer disease, rendering the infiltration in the mitral ring inoperable. Therefore, it was decided to maintain chemotherapy with ifosfamide to control the underlying disease. A second line of treatment has not been proposed so far due to an episode of febrile neutropenia with sepsis. Heart transplantation, in turn, was not indicated due to active neoplastic disease.

Despite the failure to convert the residual disease into operable, the patient has been evolving in good general condition, is asymptomatic, and is maintaining a good quality of life, with 7 months of current survival. For the follow-up, although the magnetic resonance imaging exam (MRI) had been recommended, the fact that the service in question did not perform the referred exam led to the consideration of only the transthoracic echocardiogram to monitor the case. A new surgical approach is still under discussion, especially if a reduction in tumor size from second-line drugs is considered.

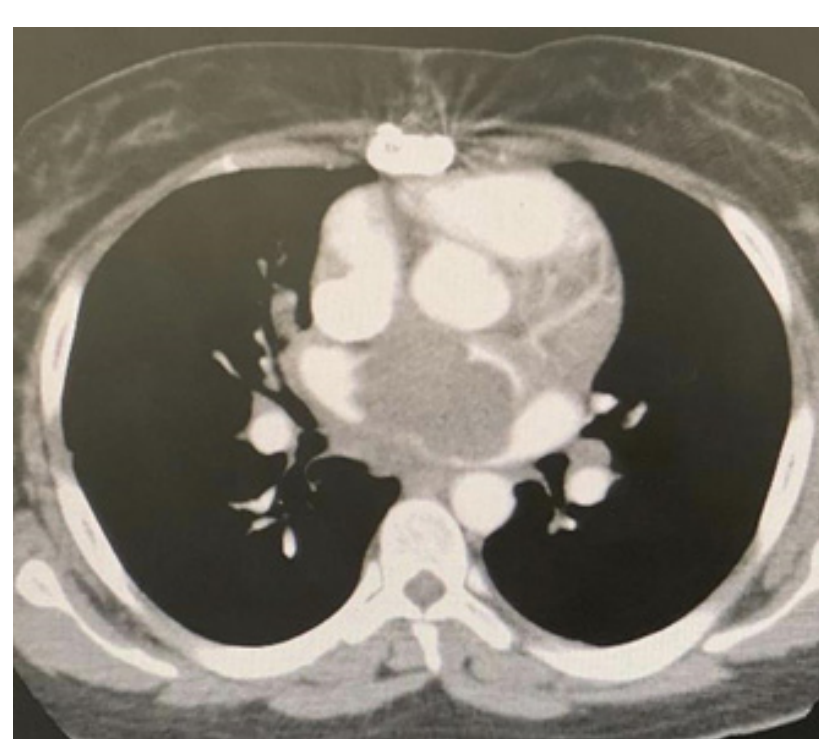

Figure 1 - Computed Tomography Exam showing heart region before chemotherapy 


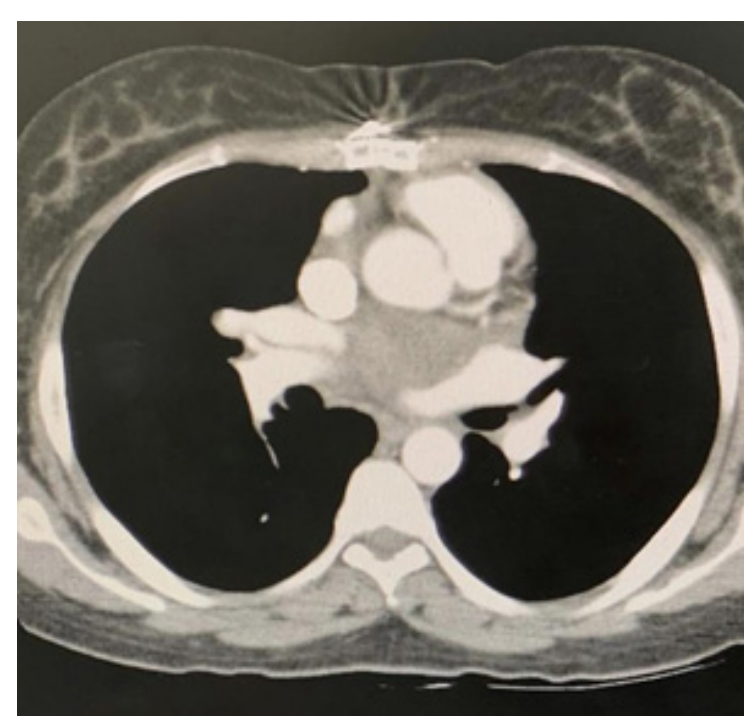

Figure 2 - Computed Tomography Exam after third cycle of chemotherapy

\section{Discussion}

Tumors of the heart and pericardial sac can be divided into primary and secondary tumors. The main histological types of primary heart tumors are myxomas, fibromyxomas, and sarcomas, which are differentiated into benign or malignant, as shown in Table 1. The main secondary tumor, in turn, comes from metastases of melanomas. ${ }^{8}$

The most common type of benign primary heart tumor is myxoma. This prevails among women of 40 and 50 years of age, and is usually located in the left atrium, close to the mitral valve leaflets. ${ }^{8}$

In its morphology, the myxoma is mostly pedicled. It is able to suffer prolapse through the mitral valve and prevent the filling of the ventricle during the diastole phase. ${ }^{9}$ In microscopic histopathological analysis, myxomas may present stellate, fusiform or elongated, polygonal cells, wrapped in a myxoid stroma. ${ }^{10}$ In addition, it has characteristics of mesenchymal cells with intermediate filaments and narrow intracellular junctions, there are no desmosomes, and some cells may have characteristics of myofibroblasts. ${ }^{10}$ Macroscopically, these can reach up to $15 \mathrm{~cm}$ and be of different colors and consistencies, in addition to being able to present a myxoid or gelatinous structure. ${ }^{9}$

The most frequent primary malignant tumor of the heart is the sarcoma type ${ }^{11}$ of the heart muscle, with a higher frequency between the third and fifth decade of life and with a predominant location in the right atrium. ${ }^{12}$ The angiosarcoma type is the most common, with a higher incidence in men, originating from mesenchymal tissue, with a broad multilobulated base, measuring between 2 and $10 \mathrm{~cm}$, dark brown or blackish in color, close to the inferior vena cava, and with early metastases to the pleura, mediastinum, and lungs. ${ }^{12}$ The patient usually shows a clinical picture of heart failure and pericardial effusion. ${ }^{12}$

Histologically, angiosarcoma can present different aspects, depending on its degree of differentiation. They may contain atypical pleomorphic endothelial cells, which form papillary structures and vascular channels in well-differentiated areas, and in areas with little differentiation have fusiform anaplastic cells. ${ }^{13}$ An angiosarcoma can, therefore, have both welldifferentiated and undifferentiated regions. ${ }^{13}$

The UPS, or malignant fibrous histiocytoma, ${ }^{14}$ in turn, makes up to one third of all cardiac sarcomas, ${ }^{15}$ and its etiology and pathogenesis are uncertain. ${ }^{16}$ The age of onset varies from 14 to 80 years of age, ${ }^{17} 47$ years of age on average. ${ }^{18}$ The incidence is higher (67\%) in women. ${ }^{17}$ Symptoms are variable and dependent on the location and size of the tumor, but dyspnea was reported in $70 \%$ of all cases; palpitations in $45 \%{ }^{17}$ chest pain in $20 \% ; 11,19$ and cough, bilateral edema of the lower limbs, and hemoptysis in $8 \%,{ }^{19}$ with nausea and vomiting also reported. ${ }^{20}$ Pericardial effusion is a complication present mainly in metastatic tumors to 
Table 1 - Differences between benign and malignant primary tumors of the heart

\begin{tabular}{|c|c|c|}
\hline & Benign tumors & Malignant tumors \\
\hline & Myxoma & Rhabdomyosarcoma \\
\hline & Rhabdomyoma & Mesenchymoma \\
\hline \multirow[t]{4}{*}{ Types } & Fibroma & Angiosarcoma \\
\hline & Lipoma & Spindle cell tumor \\
\hline & Angioma & Lymphosarcoma \\
\hline & $\begin{array}{l}\text { Papillary tumors of } \\
\text { the heart }\end{array}$ & Malignant hemangioma of the heart \\
\hline Most common type & Myxoma & Angiosarcoma \\
\hline Population with higher incidence & $\begin{array}{l}\text { Women between } 40 \text { and } 50 \\
\text { years of age }\end{array}$ & Men between 30 and 50 years of age \\
\hline Most common location & Left atrium & Right atrium \\
\hline Microscopicallly & $\begin{array}{l}\text { Starred cells, fusiform or elongated, polygonal cell, } \\
\text { wrapped in a myxoid stroma; } \\
\text { absence of desmosome }\end{array}$ & $\begin{array}{l}\text { Atypical pleomorphic endothelial cells } \\
\text { in well-differentiated areas and fusiform } \\
\text { anaplastic cells in poorly differentiated areas }\end{array}$ \\
\hline Macroscopically & $\begin{array}{c}\text { Reach up to } 15 \mathrm{~cm} \text {, varying colors and consistency; } \\
\text { present a myxoid or gelatinous structure }\end{array}$ & $\begin{array}{l}\text { Measure between } 2 \text { and } 10 \mathrm{~cm} \text {, colored black } \\
\text { or dark brown, wide and multilobulated base }\end{array}$ \\
\hline Most affected structures & Mitral valve leaflets & $\begin{array}{c}\text { Inferior vena cava, metastases to the pleura, } \\
\text { mediastinum, and lungs }\end{array}$ \\
\hline
\end{tabular}

Clinical findings

Heart failure, dyspnea, palpitations

Heart failure, pericardial effusion

Both can manifest with disorders in the electrical conduction of the heart, atrial fibrillation and flutter, ventricular extrasystoles, right bundle branch block, and, primarily, arrhythmias.

Source: Authors themselves, 2020.

the heart, which occur mainly in the pericardium, due to lymphatic dissemination, and in the right cavities, caused by hematogenous dissemination. Thus, patients with metastasis to the pericardium may present massive pericardial effusions and cardiac tamponade. ${ }^{8}$

Physiologically, patients who have benign or malignant primary tumors or secondary tumors due to metastasis from another tumor, may manifest disturbances in the electrical conduction of the heart, atrial fibrillation and flutter, ventricular extrasystoles, right branch block, and, primarily, arrhythmias. ${ }^{12}$ In addition, they may show signs and symptoms of heart failure, requiring tests to make a differential diagnosis with other pathologies. ${ }^{12}$

The best exam for an initial assessment in clinical investigation is transthoracic echocardiography. ${ }^{21}$ MRI and CT demonstrate the extent of the tumor in the myocardium and the presence of metastases. ${ }^{22}$ The diagnosis of sarcoma is only made after histological analysis, but there are characteristics of radiological tests to differentiate it from the myxoma, such as the non-septal connection of the mass, multiple masses, extensive connection to the left atrial wall, extension 
to the pulmonary vein, and semi-solid consistency. ${ }^{23}$ Moreover, gastrointestinal symptoms appear more in UPS than in myxomas. ${ }^{14}$

In general, the prognosis of tumors in the right atrium is worse, with significantly reduced survival. ${ }^{17,24}$ However, this occurs in only $7 \%$ to $10 \%$ of the UPS, ${ }^{11,17}$ which have a preference for the left atrium ${ }^{20}$ in $65.5 \%$ of cases, usually in the posterior wall, ${ }^{18}$ with an invasion of the mitral valve in up to $10 \% .{ }^{17}$ When a malignant tumor is found in the left atrium, the UPS and leiomyosarcoma are more likely, making up $44 \%$ and $22 \%$ of the cases, respectively. ${ }^{11}$

Surgery, the best treatment for local sarcomas, ${ }^{11}$ can relieve symptoms, ${ }^{25}$ but it is not usually curative, unlike that which happens with benign neoplasias. ${ }^{3}$ The prognosis is better when the tumor is completely removed, with an average survival of 4 years more than that of incomplete resection ${ }^{26}$ Although complete resection with negative microscopic margins does not prevent recurrence in $2 / 3$ of the patients, it does increase survival by 30 months, when compared to those with positive margins. ${ }^{26}$ However, the rate of complete resection is only $13 \%,{ }^{19}$ partly attributed to the difficulty in diagnosis. ${ }^{27}$ When complete resection is not practicable, more than $90 \%$ of the patients die within a year, regardless of post-surgical therapy ${ }^{15}$

The recommendation for adjuvant treatment is not well established in the literature, and there is no consensus on the ideal time for onset or duration. ${ }^{27}$ There are reports of good evolution without it, even with positive margins on microscopy after resection. ${ }^{27}$ However, there are studies that demonstrate the advantage of multimodal treatment, ${ }^{28,29}$ although the use is limited in those cases with incomplete resection. ${ }^{28}$

In one cohort, patients who received chemotherapy alone, or in combination with surgery, presented better survival rates than did those who did not receive it. However, those who received chemotherapy showed better physical performance. ${ }^{11}$ Local disease control, associated with imp17, roved cardiac function, most likely leads to greater tolerance for aggressive therapies. ${ }^{11}$

Several studies show better disease-free survival with doxorubicin without impacting overall survival. ${ }^{30}$ The usual administration is six cycles of ifosfamide and doxorubicin, ${ }^{17,20,31}$ and a better survival rate with taxanes has recently been reported. ${ }^{19}$ Sarcomas are resistant to radiotherapy. ${ }^{32}$ However, it can be used to prevent or delay local recurrence. ${ }^{26,14}$ Proton beam irradiation has been successfully used. ${ }^{28}$ Furthermore, neoadjuvant chemotherapy can increase the chance of a complete resection of the tumor, which would translate into better patient survival. ${ }^{33}$

Heart transplantation has been reported with variable results. However, this practice is hampered by the scarcity of donors, the possibility of tumor recurrence by post-transplant immunomodulation, and the exclusion of many patients for other chronic and psychosocial diseases. ${ }^{27} \mathrm{~A}$ promising alternative pointed out in the literature would be the genetic identification of the tumor for individualized therapeutic options, including tyrosine kinase inhibitors, monoclonal antibodies, and CKD-4 inhibitors. ${ }^{33}$

\section{Conclusion}

Cardiac UPS is rare and aggressive, and presents a poor prognosis, with an average survival of less than one year. It presents variable symptoms that depend on the location and can cause multiple complications. The most effective treatment for localized tumors is surgical resection, and adjuvant therapies are not well established in the literature. However, there is a consensus on the administration of six cycles of ifosfamide and doxorubicin, a protocol followed by our patient, who remained stable, with a short follow-up of only seven months.

\section{Author Contributions}

Conception and design of the research: Razera $R$, Bernardes V, Moisés F, Araújo A, Araújo R. Acquisition of data: Razera R, Bernardes V, Moisés F, Araújo A. Analysis and interpretation of the data: Razera $R$, Bernardes V, Moisés F, Araújo A, Mundim L. Writing of the manuscript: Razera R, Bernardes V, Moisés F, Araújo. Critical revision of the manuscript for intellectual content: Razera R, Bernardes V, Moisés F, Araújo A, Mundim L, Araújo R.

\section{Potential Conflict of Interest}

No potential conflict of interest relevant to this article was reported.

\section{Sources of Funding}

There were no external funding sources for this study. 


\section{Study Association}

This study is not associated with any thesis or dissertation work.

\section{Ethics Approval and Consent to Participate}

This study was approved by the Ethics Committee of the Universidade Federal de Uberlândia under the protocol number 4.041.793. All the procedures in this study were in accordance with the 1975 Helsinki Declaration, updated in 2013. Informed consent was obtained from all participants included in the study.

18. Okamoto K, Kato S, Katsuki S, Wada Y, Toyozumi Y, Morimatsu M, et al. Malignant fibrous histiocytoma of the heart: case report and review of 46 cases in the literature. Intern Med. 2001 Dec;40(12):1222-6.

19. Isambert N, Ray-Coquard I, Italiano A, Rios M, Kerbrat P, Gauthier M, et.al. Primary cardiac sarcomas: a retrospective study of the French Sarcoma Group. Eur J Cancer. 2014 Jan;50(1):128-36.

20. Balaceanu A, Mateescu D, Diaconu C, Sarsan A. Primary malignant fibrous histiocytoma of the right ventricle. J Ultrasound Med. 2010;29(4):655-8.

21. Araoz PA, Eklund HE, Welch TJ, Breen JF. CT and MR imaging of primary cardiac malignancies. Radiographics. 1999;19(6):1421-34.

22. Eswaran P, Devadoss P, Narasimhan LS, Kannan K. Synovial sarcoma of the heart: a case report and literature review. J Can Res Ther. 2015;11(3):659.

23. Kim JT, Baek WK, Kim KH, Yoon YH, Kim DH, Lim HK. A primary cardiac sarcoma preoperatively presented as a benign left atrial myxoma. Yonsei Med J. 2003;44(3):530-3.

24. Aksu T, Gode S, Oz K, Ersoy B, Ustunısık CT, Guner Y, et al. Rapidly progressive malignant fibrous histiocytoma of right atrium: a rare case report. Braz J Cardiovasc Surg. 2019;34(3):372-6.

25. Saraiva J, Antunes PE, Carvalho L, Antunes MJ. Primary malignan cardiac tumors: surgical results. Rev Port Cardiol. 2016;35(4):199-204.

26. Li H, Xu D, Chen Z, Ding W, Hong T, Chen H, et.al. Prognostic analysis for survival after resections of localized primary cardiac sarcomas: a single-institution experience. Ann Thorac Surg. 2014;97(4):1379-85.

27. Beller JP, Maddalo S, Zamuco R, Axel L, DeAnda A, Balsam LB. Right ventricular undifferentiated pleomorphic sarcoma: a case report. J Cardiol Cases. 2015;13(2):60-2.

28. Akishima S, Imai A, Enomoto Y, Shigeta O. Combined treatment for a large primary cardiac sarcoma: a case report. Surg Case Rep. 2019 Dec 5;5(1):97.

29. Randhawa JS, Budd GT, Randhawa M, Ahluwalia M, Jia X, Daw H, et al. Primary cardiac sarcoma: 25-year Cleveland Clinic Experience. Am J Clin Oncol. 2016;39(6):593-9.

30. Tierney JF. Adjuvant chemotherapy for localised resectable soft-tissue sarcoma of adults: meta-analysis of individual data. Sarcoma Metaanalysis Collaboration. Lancet. 1997;350(9092):1647-54.

31. Lennerz C, O'Connor M, Schunkert H, Deutsch MA. A case report of primary cardiac sarcoma: a diagnostic and therapeutic challenge. Eur Heart J Case Rep. 2018;2(4):143.

32. Gupta A. Primary cardiac sarcomas. Expert Rev Cardiovasc Ther 2008;6(10):1295-7.

33. Saleh WKA, Ramlawi B, Shapira OM, Jabbari OA, Ravi V, Benjamin $\mathrm{R}$, et. al. Improved outcomes with the evolution of a neoadjuvant chemotherapy approach to right heart sarcoma. Ann Thorac Surg. 2017;104(1):90-6 valve: a case report and review of the literature. J Med Case Rep. 2015 Oct 31;9:246. 